\title{
Efectos de aplicación de ácido fosfórico y microarenado en adhesivos autograbadores de uno y dos pasos
}

\author{
Alba Lucía Gómez-Carreño, Esp. (c), Laura Isabel Prada-Espinosa, Esp. (c), \\ Henry Alexander Barrios-Bastidas, Esp. (c), Mauricio Peña-Castillo, Esp. ${ }_{1}$, \\ Edgar Alexander López-López, Esp. MsC. (c) , Osnara María Monguel- \\ Gómez, MsC. PhD. ${ }_{1}$, John Alexis Domínguez ${ }^{*}, \mathrm{MsC}$. PhD. (c)
}

Recibido: 25 de noviembre del 2014. Aprobado: 29 de enero del 2015.

*Autor de correspondencia: John Alexis Domínguez. Universidad Estatal de Ponta Grossa, Ponta Grossa, Paraná, Brasil. Alameda Nabuco de Araujo 422, Barrio Uvaranas, CEP: 84031510. Teléfono 554298280372. Correo electrónico: johnalexis.dominguez@gmail.com.

Cómo citar este artículo: Gómez-Carreño AL, Prada-Espinosa LI, Barrios-Bastidas HA, Peña-Castillo M, López-López EA Monguel-Gómez OM, et al. Efectos de aplicación de ácido fosfórico y microarenado en adhesivos autograbadores de uno y dos pasos. Rev Nac Odontol. 2015;11(20):7-12. doi: http://dx.doi.org/10.16925/od.v11i20.763

Resumen. Introducción: el objetivo de esta investigación fue evaluar la resistencia de unión a las fuerzas de microcizallamiento de dos adhesivos de autograbado: un solo paso y dos pasos en esmalte, previa aplicación de ácido y microarenado. Métodos: se utilizaron 40 terceros molares humanos, recientemente extraídos, fueron lijados con 600-2500 y divididos en dos grupos: 1$)$ un paso $(n=20), 2)$ dos pasos $(n=20)$; a su vez, se subdividen en cuatro subgrupos, según el procedimiento (sA-SM: aplicación de adhesivo según fabricante, sA-M: previa aplicación de microarenado y adhesivo, CA-SM: aplicación de ácido fosfórico 37\% y aplicación de adhesivo, CA-M: aplicación de microarenado, ácido fosfórico 37\% y adhesivo). Posteriormente, fueron colocados de dos a tres cuerpos de tygon en cada superficie. Fue llevado a test de microcizallamiento. Los datos fueron analizados en test de normalidad D’Agostino. Después, se realizó Anova de dos criterios y postest Bonferrini $(\alpha=0,05)$. Resultados: para el adhesivo de dos pasos previa aplicación de ácido fosfórico 37\% o microarenado, no presenta aumento significativo de los valores de resistencia de unión en esmalte $(p=0,678)$. La aplicación de ácido fosfórico al 37\% en esmalte previo al adhesivo un paso presenta aumento de los valores de resistencia de unión $(p=0,001)$. Conclusiones: el microarenado previo al proceso de adhesión no ayuda a aumentar los valores de resistencia de unión al esmalte; la aplicación de ácido fosfórico ayuda al aumento de los valores de resistencia de unión en esmalte.

Palabras clave: adhesivos autograbados, esmalte dental, microcizallamiento, resistencia de unión. 


\title{
Effects of Phosphoric Acid and Microsandblasting in One-Step or Two-Step Self-Etching Adhesives
}

\begin{abstract}
Introduction: the object of this research was to evaluate the Microshear strength of two self-etching adhesives: one-step and two-step on enamel, upon acid and microsandblasting. Methods: 40 recently pulled human third molars, which were filed down with 600-2500 and divided into two groups: 1$)$ one step $(n=20) ; 2)$ two steps $(n=20)$; then they were divided into four subgroups, following the procedures (SA-SM: adhesive application according to the manufacturer; SA-M: upon microsandblasting and adhesive application; CA-SM: 37\% phosphoric acid application and adhesive application; CA-M: microsandblasting, 37\% phosphoric acid and adhesive). Then, three layers of tygon were applied on each surface. Microshearing test conducted. The data were analyzed with the D'Agostino normality test. Afterwards, the two-criteria Anova analysis was conducted and Bonferrini postest $(\alpha=0.05)$. Results: for the two-step adhesive prior application of $37 \%$ phosphoric acid and/or microsandblasting, there is no significant increase in the enamel adhesion strength values $(p=0,678)$. The application of $37 \%$ phosphoric acid on enamel before the one-step adhesive increases the strength values $(p=0,001)$. Conclusions: microsandblasting before the adhesive process does not help increase the enamel adhesion strength values; the application of phosphoric acid helps increase the enamel adhesion strength values.
\end{abstract}

Keywords: adhesion strength, tooth enamel, Microshear, self-etching adhesives.

\section{Efeitos da aplicação de ácido fosfórico e microjato em adesivos autocondicionantes de um e dois passos}

Resumo. Introdução: o escopo desta investigação foi avaliar a resistência de união às forças de microcisalhamento de dois adesivos autocondicionantes: um passo e dois passos em esmalte, prévia aplicação de ácido e microjato. Métodos: foram utilizados 40 terceiros molares humanos, retirados recentemente, foram lixados com 600-2500 e divididos em dois grupos: 1) um passo $(n=20), 2)$ dois passos $(n=20)$; por sua vez, foram subdivididos em quatro subgrupos, de acordo com o procedimento (SA-SM: aplicação de adesivo de acordo com orientações do fabricante, sA-M: prévia aplicação de microjato e adesivo, CA-SM: aplicação de ácido fosfórico 37\% e aplicação de adesivo, CA-M: aplicação de microjato, ácido fosfórico $37 \%$ e adesivo). Em seguida, foram colocados de dois a três tubos de Tygon em cada superfície. Foi levado teste de microcisalhamento. Os dados foram analisados em teste de normalidade D’Agostino. Depois, foi feita Anova de dois critérios e depois teste de Bonferrini $(\alpha=0,05)$. Resultados: para o adesivo de dois passos prévia aplicação de ácido fosfórico $37 \%$ e/ ou microjato, não apresenta aumento significativo dos valores de resistência de união em esmalte $(p=0,678)$. A aplicação de ácido fosfórico a 37\% em esmalte prévio ao adesivo de um passo apresenta um aumento dos valores de resistência de união $(p=0,001)$. Conclusões: o microjato antes do processo de adesão não ajuda a aumentar os valores de resistência de união ao esmalte; a aplicação de ácido fosfórico ajuda ao aumento dos valores de resistência de união em esmalte.

Palavras-chave: adesivos autocondicionantes, esmalte dentário, microcisalhamento, resistência de união. 


\section{Introducción}

Los sistemas adhesivos actuales se pueden clasificar según la utilización de ácido fosfórico (convencionales) o la no utilización (autograbado), y además, se subdividen según el número de pasos que se realizan clínicamente para su aplicación: tres pasos, cuando se presenta por separado (el ácido, el primer y el adhesivo); dos pasos, cuando el ácido y el primer se encuentran en un solo frasco y el adhesivo en otro; y un paso, cuando se encuentran los tres elementos en un solo frasco $[1,2]$. Los sistemas adhesivos autograbadores ofrecen una reducción en los pasos operatorios al suprimir el acondicionamiento previo con ácido fosfórico por la incorporación de monómeros ácidos en su composición, esto ha generado una disminución en los valores de resistencia de unión al esmalte [3].

Algunos autores hablan de realizar, con adhesivos autograbadores, un previo grabado con ácido fosfórico $37 \%$ en esmalte para proporcionar mejores valores de resistencia de unión, como los encontrados por Taschner et al. [4]. Estos autores, entre sus resultados, observaron que la resistencia de unión en adhesivo Adper Easy Bond (3M Espe) y iBond (iBond SE; Heraeus Kulzer), seis meses después de su aplicación, previo grabado con ácido fosfórico, obtuvo mejores resultados de resistencia de unión, y evaluaron adhesivos de un paso: encontraron, con previo grabado ácido, mejores valores de resistencia de unión [4].

Otros autores proponen, generando también controversias, la realización de microarenado antes del proceso de adhesión. Sohrabi et al. [5] evaluaron diferentes sistemas de adhesivos autograbadores, con resultados favorables y desfavorables según el tipo de adhesivo autograbador.

Debido a lo anterior, se presenta el siguiente objetivo: evaluar la resistencia de unión al microcizallamiento de dos adhesivos autograbadores de un paso y dos pasos en esmalte, previa aplicación de ácido y microarenado.

\section{Métodos}

Se utilizaron 40 terceros molares humanos, recientemente extraídos, libres de caries, obtenidos de pacientes que asistieron a consulta de odontología en consultorios particulares de Asunción (Uruguay); se les pidió firmar consentimiento informado, previa aceptación por comité de ética de la ciudad con protocolo (000013-0005/5). Se limpiaron las raíces de los dientes con cureta de gracey (Hu-friedy, Chicago, Illinois, EE. UU.) y fueron conservadas en agua destilada a una temperatura de $5{ }^{\circ} \mathrm{C}$ hasta el momento de su utilización.

\section{Preparación de muestra de esmalte}

Se retiró la porción radicular $1 \mathrm{~mm}$ por debajo de la unión amelocementaria en sentido vestíbulo-palatino, paralelas al plano oclusal, utilizando un micromotor (Nsk, Nakanishi Dental Spain, Tokio, Japón) de baja velocidad. Los cortes se hicieron con discos diamantados de acero (diámetro de 22 $\mathrm{mm}$, espesor de 0,15 mm, KG Sorensen, São Paulo, Brazil) y abundante agua. Posterior a esto, se lijó el esmalte empezando desde la lija de agua (Abracol, Medellín, Antioquia, Colombia) de calibre 600 hasta la lija con granulación 2500. Luego, las muestras fueron colocadas en tubos de policloruro de vinilo (PvC) (Pavco s. A., Bogotá, Cundinamarca, Colombia) con la porción lijada adherida a una cinta doble faz (Scotch 3M, Chicago, Illinois, EE. uU.). La porción no adherida se embebe en acrílico de autocurado (Veracril ${ }^{\circledR}$ Autopolimerizable, New Stetic s. A., Guarne, Antioquia, Colombia). La superficie vestibular quedó libre y preparada para realizar la colocación de tygon.

\section{División de grupos}

Las muestras obtenidas se clasificaron aleatoriamente en dos grupos según el tipo de adhesivo utilizado:

1. Adhese (Ivoclar Vivadent AG, Liechtenstein, Alemania). Dos pasos.

2. ScotchBond Universal Adhesive, 3M Espe, Neuss, Alemania). Un paso (tabla 1). 
Tabla 1. Adhesivos utilizados, lote y composición

\begin{tabular}{|l|l|l|l|}
\hline \multicolumn{1}{|c|}{ Adhesivo } & \multicolumn{1}{c|}{ Empresa } & \multicolumn{1}{c|}{ Lote } & \multicolumn{1}{c|}{ Composición } \\
\hline AdhesE & $\begin{array}{l}\text { Ivoclar Vivadent } \\
\text { AG, FL 9494, } \\
\text { Schaan, } \\
\text { Liechtenstein. }\end{array}$ & N. ${ }^{\circ}$ R76577 & $\begin{array}{l}\text { AdhesE Primer: dimetacrilato, acrilato, ácido fosfórico, ini- } \\
\text { ciadores y estabilizadores en solución acuosa. AdhesE Bond: } \\
\text { HEMA, dimetacrilato, dióxido de silicio, iniciadores y estabiliza- } \\
\text { dores. }\end{array}$ \\
\hline Universal Single Bond & $\begin{array}{l}\text { 3M Espe AG, } \\
\text { Seefeld, Alemania. }\end{array}$ & N. ${ }^{\circ} 499405$ & $\begin{array}{l}\text { MDP monómero de fosfato, dimetacrilato, HEMA, carga, etanol, } \\
\text { agua, iniciadores, silano. }\end{array}$ \\
\hline
\end{tabular}

Fuente: elaboración propia

Cada adhesivo fue conformado aleatoriamente por 20 coronas, utilizando la superficie vestibular y la superficie palatina. El total fue de 40 coronas en la superficie para realizar los procedimientos experimentales, y estos a su vez se subdividieron en cuatro grupos según el procedimiento previo a la colocación del tygon (tabla 2).

Tabla 2. División de grupos y procedimientos de cada uno de los adhesivos

\begin{tabular}{|c|c|c|l|}
\hline \multicolumn{2}{|c|}{ Grupos } & $\mathbf{n}$ & \multicolumn{1}{c|}{ Procedimientos } \\
\hline \multirow{2}{*}{ SA } & SM & 10 & $\begin{array}{l}\text { Se aplica adhesivo sobre el esmalte }(\mathrm{sF}), \text { se airea por } 5 \mathrm{~s} \text {, se fotocura por 40 s, se coloca cilindro de } \\
\text { resina de } 1 \mathrm{~mm} \text { de diámetro, se fotocura por 40 s. }\end{array}$ \\
\cline { 2 - 5 } & M & 10 & $\begin{array}{l}\text { Se realiza microarenado del esmalte por } 20 \mathrm{~s} \text { a } 10 \mathrm{~cm} \text { de distancia con óxido de aluminio de } 50 \mu \mathrm{m}, \\
\text { luego se limpia y se realiza el procedimiento del grupo sm. }\end{array}$ \\
\hline \multirow{2}{*}{ CA } & SM & 10 & Se graba el esmalte por 30 s, se lava con abundante agua y se realiza procedimiento del grupo sM. \\
\cline { 2 - 5 } & M & 10 & $\begin{array}{l}\text { Se realiza microarenado del esmalte por 20 s a 10 cm de distancia con óxido de aluminio de } 50 \mu \mathrm{mm}, \\
\text { luego se limpia, se graba el esmalte por 30 s, se lava con abundante agua y se realiza procedimiento del } \\
\text { grupo sm. }\end{array}$ \\
\hline
\end{tabular}

SA: sin ácido, CA: con ácido, SM: sin microarenar, M: con microarenado, SF: según fabricante

Fuente: elaboración propia

\section{Adhesión tygon}

Se recortó con bisturí Dimeda (Dimeda, Bogotá, Cundinamarca, Colombia), con una hoja n..$^{\circ} 15$ (Paramount Surgimed Ltda. L.s.c, Okhla Industrial Area, phase II, Nueva Delhi, India), el tygon (Tygon Tubing - 1/2 ID) de 0,6 $\mathrm{mm}$ de diámetro por $1 \mathrm{~mm}$ de altura, en donde se condensó una resina Filtex Supreme хт 350 (3M Espe, Dental Products, St. Paul, Minnesota, EE. UU.). Con una espátula metálica (Marthe, Bucaramanga, Santander, Colombia), se observa que esté sin burbujas y se posiciona el esmalte con previo tratamiento según su grupo de trabajo. Se realiza el proceso de fotoactivación con lámpara LED (Radii-cal, Southern Dental Industries, EE. UU.), cada uno por $20 \mathrm{~s}$, se separa el tygon de cada cilindro de resina con un bisturí n. ${ }^{\circ} 15$ (Paramount Surgimed Ltda. L.s.c, Okhla Industrial Area, phase II, Nueva Delhi, India) y se ubican de cinco a seis cilindros de resina sobre el esmalte de la cara vestibular de los terceros molares.

\section{Microcizallamiento}

Se llevaron los cuerpos de prueba a una máquina de ensayos universal (Kratos Dinamómetros, Embu, sP, Brazil), en la Universidad Estatal de Ponta Grossa (Paraná, Brasil). Se colocó el cincel lo más próximo a la interfase adhesiva y se hizo una carga a una velocidad de $1,0 \mathrm{~mm} /$ minuto, hasta obtener fractura de ella; los datos de resistencia de unión fueron dados en megapascales (MPa).

\section{Estadística}

Se hizo un test de normalidad D'Agostino, y después se realizó Anova de dos criterios y postest Bonferrini $(\alpha=0,05)$.

\section{Resultados}

Los valores de media y desviación estándar se presentan en la tabla 3 expresados en MPa. 
Tabla 3. Media y desviación estándar, resistencia de unión al microcizallamiento (MPA), dos adhesivos y grupos experimentales

\begin{tabular}{|c|c|c|c|c|}
\cline { 2 - 5 } \multicolumn{1}{c|}{} & \multicolumn{2}{c|}{ SA } & SM & MA \\
\cline { 2 - 5 } & SM & M & $8,79 \pm 2,45 \mathrm{~B}$ & $14,7 \pm 2,21 \mathrm{~A}$ \\
\hline Adhese & $16,3 \pm 4,37 \mathrm{~A}$ & $12,1 \pm 1,95 \mathrm{~B}$ & $36,4 \pm 3,21 \mathrm{~A}$ & $12,7 \pm 1,85 \mathrm{~B}$ \\
\hline Universal & $14,9 \pm 2,49 \mathrm{~B}$ & $13,4 \pm 1,69 \mathrm{~B}$ & \\
\hline
\end{tabular}

Letras diferentes denotan diferencia significativa entre los datos de forma horizontal

Fuente: elaboración propia

Para el adhesivo Adhese (Ivoclar Vivadent AG, Liechtenstein, Alemania), la aplicación de ácido fosfórico $37 \%$ o microarenado no presenta aumento significativo de los valores de resistencia de unión en esmalte $(p=0,678)$.

La aplicación de ácido fosfórico al 37\% en esmalte previo al adhesivo (ScotchBond Universal Adhesive, 3M Espe, Neuss, Alemania) presenta aumento de los valores de resistencia de unión $(p=0,001)$.

\section{Discusión}

Estudios hechos por Prati et al. [6] demostraron que los sistemas de grabado con ácido fosfórico muestran mayor resistencia de unión al esmalte que los adhesivos autograbadores, por lo cual es necesario conseguir una técnica que mejore la interacción entre las moléculas ácidas de los autograbadores con el sustrato del esmalte, para lograr un mayor contacto; una de estas técnicas es la aplicación activa [7].

Estudios han manifestado que sistemas autograbadores más ácidos han sido capaces de producir un patrón muy definido de grabado del esmalte, similar al ácido fosfórico $[8,9]$. Otros estudios no encuentran diferencia significativa, razón por la cual se sugiere la aplicación previa de ácido fosfórico [10]. En este estudio, aunque no se evaluaron los valores de resistencia de unión entre los adhesivos, la diferencia entre ellos puede explicarse por estos motivos. La concentración y el tipo de monómeros ácidos que alteran directamente la acidez de los sistemas adhesivos de autograbado, pueden contribuir a la disociación parcial o total para el grabado en esmalte [11].
Algunos estudios que emplearon microscopía electrónica concluyeron que, en el esmalte tratado con sistemas autograbadores, fue descalcificado selectivamente el esmalte interprismático, y mostró tags delgados con una estructura porosa a diferencia de los tags gruesos que penetran en el esmalte tratado con ácido fosfórico. Debido a esto, indicaron que el ácido fosfórico provoca una superficie ideal en el esmalte para la adhesión, contrario a los monómeros ácidos incorporados en los adhesivos autograbadores que presentan insuficiencia [12]. Por tanto, como dice el estudio de Van Landuyt et al. [13], encontraron que la utilización de ácido fosfórico antes de la aplicación de los autograbadores mejora la efectividad adhesiva, pero debe limitarse al esmalte. En el estudio de Poggio et al. [14], en el pretratamiento del esmalte con ácido fosfórico, se obtuvo un aumento significativo de los valores de resistencia de unión de todos los adhesivos probados.

En este estudio fueron evaluados dos adhesivos autograbadores de uno y dos pasos: Adhese (Ivoclar Vivadent AG, Liechtenstein, Alemania) de dos pasos presenta un pH 3,4 [15], y el adhesivo ScotchBond Universal Adhesive (3M Espe, Neuss, Alemania) además presenta MDP (monómeros fosfatados) [16], elemento que ayuda a mejorar la adhesión química. Esta es una de las razones por las cuales el adhesivo de dos pasos, cuando fue realizado el microarenado, aumentó los valores de resistencia de unión, contrario al ScotchBond Universal, ya que en este adhesivo interfiere la adhesión química.

Los resultados de este estudio sobre el microarenado son controversiales, como lo es la literatura, en la que la utilización de microarenado aumenta los valores de resistencia de unión de uno de los adhesivos (concuerda con el estudio de Wiechmann [17]), y disminuye en otro (concuerda con el estudio de Reisner [18]). 


\section{Conclusiones}

Teniendo en cuenta las limitaciones del presente trabajo, se concluye: el microarenado previo al proceso de adhesión no aumenta los valores de resistencia de unión al esmalte, y la aplicación de ácido fosfórico aumenta los valores de resistencia de unión en esmalte con la aplicación de adhesivo autograbador de un solo paso.

\section{Referencias}

[1] Perdigao J, Van Meerbeek B, Yucel T, Vanherle G. The Interaction of Adhesive System with Human Dentine. Am J Dent. 1996;9(4):167-73.

[2] Yoshiyama M, Matsuo T, Ebisu S, Pashley D. Regional Bond Strengths of Self-Etching/Self-Priming Adhesive Systems. J Dent. 1998;26(7):609-16.

[3] De Munck J, Van Landuyt K, Peumans M, Poitevin A, Lambrechts P, Braem M, et al. A Critical Review of the Durability of Adhesion to Tooth Tissue: Methods and Results. J Dent Res. 2005;84(2):11832.

[4] Taschner M, Nato F, Mazzoni A, Frankenberger R, Falconi M, Petschelt A, et al. Influence of Preliminary Etching on the Stability of Bonds Created by One-Step Self-Etch Bonding Systems. Eur J Oral Sci. 2012;120(3):239-48.

[5] Sohrabi A, Amini M, Afzali BM, Ghasemi A, Sohrabi A, Vahidpakdel SM. Microtensile Bond Strength of Self-Etch Adhesives in Different Surface Conditionings. Eur J Paediatr Dent. 2012;13(4):317-20.

[6] Prati C, Chersoni S, Ferrieri P, Mongiorgi R, Checchi L. Handling of Bonding Agents: Clinical Data. En: Dondi dall'Orologio D, Prati C, editores. Factors Influencing the Quality of Composite Restorations. Theory and Practice. Carimate: Ariesdue SRL Publishers; 1997. p. 65-83.

[7] Caneppele TM, Torres CR, Sassaki A, Valdetaro F, Fernandes RS, Prieto de Freitas C, et al. Effects of Surface Hydration State and Application Method on the Bond Strength of Self-Etching Adhesives to Cut Enamel. J Adhes Dent. 2012;14(1):25-30.
[8] Breschi L, Gobbi P, Falconi M, Mazzotti G, Prati C, Perdigão J. Ultra-morphology of Self-Etching Adhesives on Ground Enamel: A High Resolution SEM Study. Am J Dent. 2003;16 Spec:57A-62A.

[9] Hashimoto M, Ohno H, Yoshida E, Hori M, Sano H, Kaga M, et al. Resin-Enamel Bonds Made with Self-Etching Primers on Ground Enamel. Eur J Oral Sci. 2003;111(5):447-53.

[10] Pashley DH, Tay FR. Aggressiveness of Contemporary Self-Etching Adhesives. Part II: Etching Effects on Unground Enamel. Dent Mater. 2001;17(5):430-44.

[11] Nishiyama N, Suzuki K, Takahashi K, Nemoto K. The pка Effects of the Carboxylic Acid in N-Methacryloyl-Omega-Amino Acid on the Demineralization and Bond Strengths to the Teeth. Biomaterials. 2004;25(23):5441-7.

[12] Pilecki P, Stone D, Sherriff, Watson T. Microtensile Bond Strengths to Enamel of Self-Etching and One Bottle Adhesive Systems. J Oral Rehabil. 2005;32(7):531-40.

[13] Van Landuyt KL, Kanumilli P, De Munck J, Peumans M, Lambrechts P, Van Meerbeek B. Bond Strength of a Mild Self-Etch Adhesive with and without Prior Acid-Etching. J Dent. 2006;34(1):77-85.

[14] Poggio C, Scribante A, Della Zoppa F, Colombo M, Beltrami R, Chiesa M. Shear Bond Strength of OneStep Self-Etch Adhesives to Enamel: Effect of Acid Pretreatment. Dent Traumatol. 2014;30(1):43-8.

[15] Dieng-Sarr F, Sharrock P, Dabsie F, Grégoire G. Modifications of the Organic and Mineral Fractions of Dental Tissues Following Conditioning by Self-Etching Adhesives. J Dent. 2011;39(2):141-7.

[16] Muñoz MA, Luque I, Hass V, Reis A, Loguercio $\mathrm{AD}$, Bombarda NH. Immediate Bonding Properties of Universal Adhesives to Dentine. J Dent. 2013;41(5):404-11.

[17] Wiechmann D. Lingual Orthodontics. (Part 3): Intraoral Sandblasting and Indirect Bonding. J Orofac Orthop. 2000;61(4):280-91.

[18] Reisner KR, Levitt HL, Mante F. Enamel Preparation for Orthodontic Bonding: A Comparison between the Use of a Sandblaster and Current Techniques. Am J Orthod Dentofacial Orthop. 1997;111(4):366-73. 